\section{A program to determine data transformations maximizing linear regression}

\section{WILLIAM P. DUNLAP and JEFFERY L. RASMUSSEN Tulane University, New Orleans, Louisiana 70118}

Data transformations are used to better meet the assumptions of statistical tests, to attempt to reduce interaction in data, and to produce linear relations in nonlinear data in order to better understand the underlying relationship. The present program deals with the last of these. If a linearizing transformation can be discovered for correlational data, then the back transformation can be viewed as a fitted function. Most hand-calculation procedures for such curve fitting ask the user to select a particular function to be fit, and then the data are transformed in such a way that the transformed data will be linear if the function fits. A drawback to this approach is that one minimizes the error sum of squares of the transformed, rather than the original, data. Lewis (1963) showed that the sum-ofsquares error in the back-transformed data is not the least squares minimum; thus, the best-fitting function is not usually found with this method. More recent computer-assisted curve-fitting procedures require the user to specify starting values as well as the function to be fit and then iteratively converge on the optimal values for these parameters (e.g., Dixon \& Brown, 1979, Program BMDP3R; Hochhaus, 1981). The present approach does not require the user to specify any particular function or starting values; instead, it employs a family of transformations proposed by Box and Cox (1964) that depends upon a single parameter, $\lambda$. The program then transforms one of the two variables via this transformation and iteratively converges on the value that maximizes the linear regression between the two variables. In its most sophisticated mathematical form the transformation family is $X^{*}=\left(X^{\lambda}-1\right) / \lambda$ were $X$ represents raw data and $X^{*}$ represents the transformed score. Since division by a constant and subtraction of 1 are linear transformations, they do not affect the size of the resulting correlation, thus the simpler transformation $\mathrm{X}^{*}=\mathrm{X}^{\lambda}$ is employed in the present program. When $\lambda=-1$ this function produces the reciprocal transformation, when $\lambda=0$ the logarithmic transformation, and when $\lambda=.5$ the square root; when $\lambda=1$ the data are unchanged. Therefore, the value of $\lambda$ that maximizes the correlation between the two variables can be used as a guide to the type of function that will best fit the data. This family of transformations was used previously by Dunlap and Duffy (1974) in a program that searches for transformations minimizing skew.
The program works as follows. First, the sample size and then the pairs of data points are read. Next, the data are searched for zero or negative values. If any are found, the absolute value of the minimum data value plus one is added to each score before proceeding, since at certain values of $\lambda$ the transformation is defined only for positive numbers. A scatterplot of the raw data is then depicted on the CRT screen, followed by the correlation for untransformed data. Next, the first variable is transformed to find a maximizing value for $\lambda$ in the following way: (1) A series of $\lambda$ values are tried to find the general region of best correlation. (2) The three best points are fit with a parabola to obtain an approximate maximum. (3) That approximate maximum is then substituted for the previous worst point and a new parabola is fit. (4) This process is repeated until a stable maximizing $\lambda$ is found, 50 iterations are exceeded, a parabola cannot be fit to the points, or the $\lambda$ value exceeds \pm 10 .

If a stable $\lambda$ is found, which is usually the case, a scatterplot of the transformed relationship is displayed. This scatterplot is useful in detecting possible pathological cases in which, for example, the transformation serves to concentrate all but one of the data points, thus improving the correlation artificially. The user is next given the option to write the transformed data to a file. The program then proceeds to transform the second variable relative to the untransformed first variable in the same manner as above. When the program was tested on 96 data sets $(n=100)$ with various degrees of nonlinearity, the maximizing function took an average of $734 \mathrm{msec}$ of CPU time and an average of nine iterations to converge on the correct $\lambda$ value.

Requirements. The program is written in singleprecision FORTRAN IV and runs on a DEC 2060 computer. Since the program is interactive, it is designed to use a computer terminal for much of the input and output, although data may also be read from a file. Although an effort was made to avoid DEC-specific FORTRAN statements, a few nonstandard FORTRAN statements are used to make the program nun conveniently on the DEC system. Therefore, users with other computers will probabily need to modify assignments of logical units, statements with an "*" in the usual place of a format statement number (this causes free-format input on our system), and input format statements with the final symbol "\$," which causes the cursor to remain at the end of a line on the DEC system.

Availability. A listing of the program may be obtained free of charge from William P. Dunlap, Department of Psychology, Tulane University, New Orleans, Louisiana 70118 . 


\section{REFERENCES}

Box, G. E. P., \& Cox, D. R. An analysis of transformations. Journal of the Royal Statistical Society (Series B), 1964, 26, 211-243.

Dixon, W. J., \& Brown, M. B. (Eds.). BMDP-79, biomedical computer programs, P-series. Berkeley: University of California Press, 1979.

DunlaP, W. P., \& Duffy, J. A. A computer program for determining optimal data transformations minimizing skew. Behavior Research Methods \& Instrumentation, 1974, 6, 46-48.

Hochraus, L. Curve fitting by estimation and iteration. Behavior Research Methods \& Instrumentation, 1981, 13, 753-754.

LEwIs, E. V. Statistical analysis. Princeton, N.J: Van Nostrand, 1963.

(Accepted for publication March 31, 1982.) 